Rapid Reviews COVID-19

\title{
Review 2: "College \\ campuses and COVID-19 \\ mitigation: clinical and \\ economic value"
}

\section{David Kim}

${ }^{1}$ Assistant Professor, Tufts Medical Center, Center for the Evaluation of Value and Risk in Health

Published on: Sep 30, 2020

DOI: $10.1162 / 2 \mathrm{e} 3983 f 5.57 \mathrm{~d} 7 \mathrm{bfad}$

License: Creative Commons Attribution 4.0 International License (CC-BY 4.0). 


\section{$\underline{\text { RR:C19 Evidence Scale rating by reviewer: }}$}

- Strong. The main study claims are very well-justified by the data and analytic methods used. There is little room for doubt that the study produced has very similar results and conclusions as compared with the hypothetical ideal study. The study's main claims should be considered conclusive and actionable without reservation.

$* * * * * * * * * * * * * * * * * * * * * * * * * * * * * * * * * * * * * * * *$

\section{Review:}

First of all, this is a very comprehensive modeling study of the timely and important topic of COVID-19 mitigation strategies for college campuses. Using a dynamic microsimulation model after accounting for community transmissions, the authors examined 24 different mitigation strategies, combining extensive social distancing (ESD), masks, and routine laboratory tests (RLT). Authors concluded that extensive social distancing with mandatory mask-wearing would prevent 3,253 COVID-19 cases (87\% reduction) in relative to no mitigation strategy, and were cost-effective ( $\$ 49,200 / Q A L Y)$, compared to masks alone. The evidence presented is sound and informative to help inform decisions about mitigation strategies for college campuses. Budget impact analyses and threshold analyses for the percentage of students who need to defer are good additions and highly relevant to college administrators. I have three main points to consider when understanding the findings: 1 ) be cautious about the certitude of the estimates, 2) "contact-hours" and "infectivity contract/hour" as key inputs, and 3) no consideration of "non-health impact" of the mitigation strategies.

1. I applaud the author's extensive efforts to identify and derive relevant data inputs whenever possible. Still, we should be extremely cautious about providing certitude of the estimates when we don't know much about model inputs. The analysis would greatly benefit from more extensive sensitivity analyses, ideally in the form of probabilistic analyses. At least, if authors can provide a few scenarios (e.g., optimistic, base, pessimistic scenarios), it would help to understand possible uncertainty around the estimates. Given the author's extensive evaluation of 24 strategies, it may be helpful to conduct these additional analyses, at least, for a few selected strategies in the efficiency frontiers. 
2. This comprehensive model primarily relied on "contact-hours", defined as a single hour spent with a single person. To my understanding, it means that 10 hours spent with a single person would have the same risk of 1 hour spent with 10 persons, which generally poses a different risk (much higher risk for the latter). It may not be feasible to model the differences, but it would be great to acknowledge this more explicitly. Related to the point, one of the key parameters to drive the model results would be "infectivity contact/hour". The authors derived infectivity/contact-hour based on the rate of infection among household contacts in Wuhan, China, which assumes that members of the same household spent on average 11.89 hours/day at home. Going back to the earlier point, it is unclear how this estimate is relevant to the student/faculty in the US setting. To me, this is a key input that requires careful examination through a sensitivity analysis, which was missing.

3. While the authors claimed that the study is conducted from a "modified" societal perspective, it is only so because costing for the mitigation strategy (i.e., intervention $\&$ implementation costs) does not fall on the health care sector. I would consider this as part of "intervention costs", instead of a societal perspective analysis. For the purpose of a societal perspective, the analysis would include the "non-health impact" of the intervention (e.g., lost earning or spillover effects on other sectors) in its analyses. A recent MDM letter clearly laid out this and what needs to be considered from a societal perspective analysis.

(https://journals.sagepub.com/doi/full/10.1177/0272989X20922987) Also, the authors mentioned, "Indirect costs, such as lost productivity due to isolation, were captured in utility measures". Authors need to be more explicit about the fact that this is an assumption (without supporting data), and it may only capture the "psychological" effect of less productivity. Despite some debate, recent literature supported that when people respond to the health-related quality of life utility estimation, they generally do not consider "lost productivity in terms of earnings" [see chapter 8 in the $2^{\text {nd }}$ Panel's revised report, DOI:10.1093/acprof:oso/9780190492939.003.0008]. For the primary target population (students), it may not matter, but it does for other populations (faculty or community). The authors need to be more explicit about their approach to "modified" societal perspective and possibly provide some of the implications on their estimates by adding non-health impacts.

- $\quad$ Minor points: 
o Table 1:

$\S$ Age distribution is not presented in percentage

$\S$ Health-related quality of life estimates used are not provided in Table 1 or Appendix. (Only references were provided)

$\S$ Under the intervention costs, the sum of Mask and ESD is not equal to the intervention cost of Mask + ESD. Also, these estimates are different from the text on page 12 . 